\title{
HEMICHOREA-HEMIBALLISM IN VARIOUS CONDITIONS: SERIAL CASE REPORTS
}

\author{
Neila Raisa ${ }^{1}$, Sri Budhi Rianawati ${ }^{1}$, Shahdevi Nandar Kurniawan ${ }^{1}$, Fahimma ${ }^{1}$, Mulika Ade Fitria Nikmahtustsani ${ }^{1}$ \\ Correspondence: neilaraisa@ub.ac.id \\ ${ }^{1}$ Department of Neurology Faculty of Medicine Brawijaya University, Malang, Indonesia.
}

\author{
Article History: \\ Received: July 28, 2020 \\ Accepted: July 28, 2020 \\ Published: January 1, 2021

\section{Cite this as:} \\ Raisa N, Rianawati $S B$, \\ Kurniawan SN, Fahimma, \\ Nikmatustani MAF. Hemichorea- \\ hemiballism in various \\ conditions: serial case reports. \\ Malang Neurology Journal \\ 2021.7:74-79. \\ http://dx.doi.org/10.21776/ub.mnj \\ .2021.007.01.15
}

\section{ABSTRACT :}

Introduction: Hemichorea-hemiballism (HCHB) is an uncommon movement disorder involved unilateral extremities characterized by irregular, poorly patterned, a continual hyperkinetic involuntary movement disorder in the proximal or distal parts of the body. The acute development of HCHB depends on focal lesions on the contralateral basal ganglia and subthalamic nuclei. Various conditions such as cerebrovascular, neurodegenerative, neoplastic, immunologic, infectious, and metabolic diseases are known as secondary causes of HCHB. This paper aims to compare and discuss the HCHB in various etiologies.

Case Reports: Here, we reported 5 cases of HCHB induced by non-ketotic hyperosmolar hyperglycemia (NKKH), thrombotic stroke, and toxoplasmosis cerebral. We compare the admission data, clinical course, imaging, treatment, and outcome of every case.

Conclusion: Various hypotheses have been proposed to explain the pathophysiology of HCHB due to these conditions. Principally, the main management for these cases is to determine the etiology and correct the underlying disorder.

Keywords: Hemichorea-Hemiballism, Non-Ketotic Hyperosmolar Hyperglycemia, Thrombotic Stroke, Toxoplasmosis Cerebral

\section{Introduction}

Chorea-ballism is an uncommon movement disorder characterized by irregular, poorly patterned, a continual hyperkinetic involuntary movement disorder in the proximal or distal parts, mainly involved unilateral extremities and called as hemichorea-hemiballism, but sometimes bilateral. Various conditions such as cerebrovascular, neurodegenerative, neoplastic, immunologic, infectious, and metabolic diseases are known as secondary causes of chorea-ballism. Hemichoreahemiballism (HCHB) is a rare case, even the prevalence in the population is not known clearly, but most cases have been described in individuals with Asian descent, females, and the elderly. ${ }^{1,2,3,4,5}$ Autoimmune diseases and infections often occur in HCHB at a young age, whereas in the cerebrovascular disease that has a history of hypertension and diabetes mellitus often occurs in older age. ${ }^{1,6}$

The acute development of HCHB depends on focal lesions on the contralateral basal ganglia and subthalamic nuclei. Hyperkinetic movements caused by disruption of the indirect pathway that promote a loss of inhibition on the pallidum, and excessive dopaminergic activity. It is possible to understand $\mathrm{HCHB}$ as the manifestation of various abnormalities in brain. ${ }^{5,7}$ The most common cause of HCHB due to structural lesions is a cerebrovascular disease and the most common cause of metabolic disorders is non-ketotic hyperosmolar hyperglycemia. ${ }^{8,9} \mathrm{HCHB}$ in various etiologies and characteristics is not widely reported.
We report 5 cases of $\mathrm{HCHB}$ induced by various medical abnormalities.

\section{Case Reports}

\section{First Case}

A 65-year-old woman with a history of hypertension and uncontrolled diabetes since a year ago came with the lefthand involuntary movement for two weeks before admitted to the hospital. Two weeks after, the involuntary movement progressed to her left leg. Patients felt jerking, writhing, and twisting in her left extremities and could not control the movement. The movements were painless and appear continuously and heavier when they are awake and doing some activities. However, these involuntary movements resolved during sleep.

We found involuntary and arrhythmic movements in her left extremities that appropriate as hemichoreahemiballism-like-movements. The vital sign was stable and no neurological deficit was found. Laboratory test showed random blood glucose (RBS) level was $500 \mathrm{mg} / \mathrm{dL}$, fasting blood glucose level was $225 \mathrm{mg} / \mathrm{dL}, 2$ hours postprandial blood glucose level was $452 \mathrm{mg} / \mathrm{dL}$, and with $\mathrm{HbA1C}$ 10.6\%. Electrolyte Serum showed $\mathrm{Na} 137 \mathrm{Mmol} / \mathrm{L}$ and potassium $3.45 \mathrm{Mmol} / \mathrm{L}$ with calculated serum osmolarity was $305 \mathrm{mOsm} / \mathrm{kg}$. Head CT was performed and showed hyperdense lesions on the right putamen and hypodense lesion in the left anterior periventricular region. Other pertinent admission data can be found in Table 1. We 
diagnosed the patient with $\mathrm{HCHB}$ due to the hyperglycemic state.

The involuntary movement was improved and completely resolved after she got aggressive fluid rehydration and intravenous insulin therapy. The patient discharged with basal insulin injection therapy $10 \mathrm{IU}$ and prandial insulin injection $6 \mathrm{IU}$ q8hr to control the $\mathrm{DM}$ and $1.5 \mathrm{mg}$ haloperidol PO q12hr and $2 \mathrm{mg}$ clonazepam PO q12hr to control her movement disorder.

\section{Second Case}

A 65-year-old woman with a history of hypertension since five years ago and uncontrolled DM since three years ago came to the ER complained involuntary movement of the left limb since seven days before admitted to the hospital. The patient felt uncontrolled dancing when she did an activity and disappears when she was asleep. Previously, consume 5mg Glibenclamide PO qDay but not routinely controlled.

When came to the ER, blood pressure was $160 / 70 \mathrm{mmHg}$ and no neurological deficit was found. We found involuntary jerking movements in the left extremities. RBS result was $530 \mathrm{mg} / \mathrm{dL}$, fasting blood glucose level was 210 $\mathrm{mg} / \mathrm{dL}$, postprandial blood glucose level was $228 \mathrm{mg} / \mathrm{dL}$ and $\mathrm{HbA} 1 \mathrm{C}$ was $12.3 \%$. Electrolyte Serum showed Na 133 $\mathrm{Mmol} / \mathrm{L}$ and potassium $3.4 \mathrm{Mmol} / \mathrm{L}$ with calculated serum osmolarity was $322 \mathrm{mOsm} / \mathrm{kg}$. We performed head CT and showed a hypodense lesion on left corona radiata, left lentiform nucleus, and left internal capsule posterior limb and hyperdense punctate lesions in the right-left corticalsubcortical frontal, temporal, and parietal lobes and right lentiform nucleus.

The patient was diagnosed with HCHB due to non-ketotic hyperosmolar hyperglycemia. The involuntary movement was decreased after she got fluid rehydration and intravenous continuous insulin therapy. The patient was discharged after 7 days of treatment and got basal insulin therapy $10 \mathrm{IU}$ to control DM, 0.5mg haloperidol PO q12hr, $2 \mathrm{mg}$ trihexyphenidyl PO qDay, and $1 \mathrm{mg}$ clonazepam PO $\mathrm{q} 12 \mathrm{hr}$ to control her movement disorder. Other pertinent admission data can be found in Table 1 .

\section{Third Case}

A 65-year-old woman came to the ER complained suddenly involuntary movement in her right hand which subsequently extended to her right leg when she woke up since 7 days before. This movement was painless, and no history of weakness in the limbs nor slurred speech. She had no history of the previous disease and was not taking any certain drugs.

Her condition was stable with a normal vital sign, no neurological deficit. We found involuntary movements in the right extremities irregular and continuously. Laboratory tests showed RBS was $476 \mathrm{mg} / \mathrm{dL}$, fasting blood sugar was $240 \mathrm{mg} / \mathrm{dL}$, two hours postprandial blood sugar was 263 $\mathrm{mg} / \mathrm{dL}$, and HbA1C $15.20 \%$. Urinalysis showed glucose in the urine was positive, and negative ketones. Serum electrolyte showed sodium was $133 \mathrm{Mmol} / \mathrm{L}$, potassium 4.38 Mmol/L with hyperosmolarity. We performed the head CT scan and showed hyperdense lesions in the left caudate nucleus and left lentiform nucleus which suspected as basal ganglia calcification. We diagnosed this patient with $\mathrm{HCHB}$ related to non-ketotic hyperosmolar hyperglycemia.
Her involuntary movement was resolved after we gave fluid rehydration and intravenous continuous insulin therapy and followed by oral drug Glimepiride $2 \mathrm{mg}$. Her movement disappears completely on day 4 after the treatment. She was discharged with $1 \mathrm{mg}$ glimepiride PO qDay, 500mg Metformin PO q8hr, and $2 \mathrm{mg}$ clonazepam PO qDay. The summary of the data can be found in Table 1 .

\section{Fourth Case}

A 64-year-old woman came to the ER complained involuntary movement in her left hand since 8 days ago, her movement became more intense and extended to the left leg and left face. The patient felt likes throwing and stretching irregularly movement which disappeared when she was asleep. She had a history of movement disorder with the same characteristic about 1 month ago when she had a stroke. Movement disorder occurred on the third day of hospitalization. Patients had a history of controlled hypertension with amlodipine 1 x $10 \mathrm{mg}$ and routinely controlled diabetes mellitus with insulin injection.

Her vital sign showed blood pressure 140/90 $\mathrm{mmHg}$ and heart rate $106 \mathrm{bpm}$. We found sinistra facial and hyoglossus nerve paralysis with upper motor neuron type and sinistra hemiparesis as sequelae. There were involuntary irregular stretching movements on the left part of the body which include face and extremities. On laboratory test, RBS was $117 \mathrm{mg} / \mathrm{dL}$, fasting Blood Sugar was $100 \mathrm{mg} / \mathrm{dL}$, and twohour postprandial blood sugar was $182 \mathrm{mg} / \mathrm{dL}$. Both of serum electrolyte and hemostasis was normal. Head CT scan showed sub-acute cerebral infarct at external capsules dextra and cerebellum dextra, and chronic infarct at thalamus sinistra. We diagnosed this patient with $\mathrm{HCHB}$ due to thrombotic stroke. Her involuntary movement resolved completely after 4-day treatment with $1.5 \mathrm{mg}$ haloperidol PO q8hr, 2mg clonazepam PO q12hr, levodopa PO q12hr. The summary of the data can be found in Table 1 .

\section{Fifth Case}

A 26-year-old male came to the emergency room with a gradual decrease in consciousness for 4 days before hospitalization. Previously, he complained of progressive headache, intermittent fever, and intermittent diarrhea for the last 4 months. One month before admitted to the hospital, he complained involuntary movement on his left hand, and 2 weeks later expanded to the left leg. He felt stretching and dancing movements which disappeared when he slept. He felt progressive weakness at his right side of the body. He also complained of cough without shortness of breath and mouth ulcer since 1 week ago. His family said that he never had any history of the previous disease. He consumes alcohol and had tattoos on his chest.

From the physical examination, the patient was unconscious with GCS 8/15, blood pressure was 90/50, HR was $80 \mathrm{bpm}$, respiratory rate was $22 \mathrm{tpm}$, and temperature $37.1{ }^{\circ} \mathrm{C}$. There was a mouth ulcer, the pupillary reflex was reactive with diameter $5 \mathrm{~mm}$ bilateral, we found rhonchi bilaterally. On the left extremity, there were involuntary movements with large amplitude and irregular writhing movements. We found the right lateralization at the extremities. Laboratory tests showed reactive to HIV. Complete blood count, RBS, and serum electrolyte were normal. Chest X-Ray showed pneumonia and head CT Scan 
showed rim enhanced lesion in the bilateral basal ganglia with leptomeningeal enhancement in the bilateral frontal and parietal lobes suggest as cerebral toxoplasmosis with 3 $\mathrm{mm}$ subfalcine herniation to the right of and mild communicating hydrocephalus. We diagnosed the patient with cerebral toxoplasmosis in HIV with hemichoreahemiballism complications. To reduce the involuntary movement, we gave $2 \mathrm{mg}$ clonazepam PO qDay, $1 \mathrm{mg}$ Haloperidol PO q12hr, 2mg Trihexyphenidyl PO q12hr. We treat the cerebral toxoplasmosis with pyrimethamine and clindamycin. The patient got the septic condition and passed away on the second week of treatment caused by septic encephalopathy. The summary of the data can be found in Table 1.

\section{Discussion}

Hemichorea-hemiballism (HCHB) is a hyperkinetic hypotonic movement disorder characterized by nonpatterned, jerky, random, irregular, and uncontrolled movements that occur on half parts of the body. ${ }^{1,9}$ This is due to involvement disorders at the basal ganglia, especially in the corpus striatum. ${ }^{9,10}$ The most common cause of HCHB is stroke and the second cause is non-ketotic hyperosmolar hyperglycemia (NKKH). ${ }^{6,9}$ The incidence of HCHB that occurs in the population due to post-stroke patients and NKHH is around 0.008 and $0.001 \%$. Other causes reported are due to infections, neoplasms, drug or toxins, autoimmune, and other metabolic disorders such as hyperglycemia, hypoglycemia, hypokalemia. ${ }^{6,9,11}$ In these present reports, we report 5 newly diagnosed cases HCHB due to NKKH, stroke, and toxoplasmosis cerebral.

The onset of movement disorder developments varies, depend on the cause. ${ }^{1,11}$ HCHB associated with stroke usually will suddenly appear when the paresis in the extremity has improved ${ }^{2,11}$ HCHB associated with inflammation or infection usually develops slowly, expanding, and gets heavier depends on the development of infection and inflammation. ${ }^{6,13}$ Besides, HCHB associated with cerebrovascular disease and the metabolic disorder usually occurs in older age, while in infection or inflammation occurs at a relatively younger age. ${ }^{12,13}$

The type and location of structural lesions cause diverse characteristics of movement disorder. ${ }^{14,15}$ In about $20 \%$ of cases, structural lesions that occur in HCHB takes place in the contralateral nucleus subthalamic. Besides, bilateral or ipsilateral lesions of the movement were also found in the case report caused by chronic hepatic encephalopathy. ${ }^{14,15,16,17}$

HCHB related to NKHH can be an early symptom of unknown diabetes mellitus as happened in our third patient or can appear several days or weeks when the blood glucose level of increase or is not well controlled as in our first and second patients. ${ }^{14,15}$ Several case reports showed that HCHB also can occur a few weeks after the blood glucose levels have been controlled and on treatment which shows delayed reaction to severe hyperglycemia. ${ }^{5}$ All of our NKKH patients are diagnosed as HCHB due to NKHH and were reduced or resolved perfectly after they got therapy to reduce blood glucose levels, which shows the temporal relationship between the restoration of blood glucose and the improvement of the HCHB. ${ }^{5,14,15}$ In three of our cases, that report $\mathrm{HCHB}$ caused by NKKH, the average RBS was above $400 \mathrm{mg} / \mathrm{dL}$ and the HbA1C level was above $10 \%$. HCHB has most commonly seen in patients with long-standing, poorly controlled, and often with HbA1C more than $10 \%{ }^{18}$ This also consistent with the epidemiological research in India by Nadig, et al showed that mean serum glucose levels measured after the onset of NKKH were $439.93 \mathrm{mg} \%$ and the mean $\mathrm{HbA1C}$ was $10.98 \%$. In the second and third cases, there was mild dilutional hyponatremia that suggests resulting from hyperglycemia. In acute hyperglycemia, dilutional hyponatremia can occur due to fluid transfer from cells to plasma as a result of hypertonic extracellular fluid and osmotic fluid differences. Each increase in blood glucose of $100 \mathrm{mg} / \mathrm{dl}$, serum osmolarity will increase from 1.9 to 2.1 mosm and serum sodium decreases from 1.6 to $1.8 \mathrm{mEq} / \mathrm{L}$. In chronic hyperglycemia, loss of sodium and hyponatremia can occur due to osmotic diuresis. ${ }^{14,16}$

We concluded the patient experienced HCHB associated with stroke whereas stroke is the leading cause of HCHB. ${ }^{6}$ It was supported by the imaging that showed structural lesions in the basal ganglia region at putamen and thalamus. $\mathrm{HCHB}$ associated with cerebrovascular disease or stroke can occur on acute onset after the stroke or maybe several days or months later. ${ }^{17,19}$ In this patient, HCHB first occurred on the 3rd day of the stroke, then resolved with treatment, and reoccurred when the patient did not consume medication to control HCHB during the last 2 weeks. In most cases, HCHB due to stroke can be resolved completely with appropriate medication. ${ }^{17,20}$

Movement disorders occur due to dysfunction in motor network connectivity. Connection disturbance between internal globus pallidus, subthalamic nucleus, and thalamus promotes the failure of the basal ganglia and cortical circuits. ${ }^{69}$ The distinctive characteristics of HCHB with MR and $\mathrm{CT}$ modality can exhibits lesions in the basal ganglia. $^{21,22}$

Hemichorea lesion usually find at contralateral of nucleus caudate whereas in hemiballism at the nucleus subthalamic. ${ }^{19,23}$ Although in some case reports, lesions at nucleus lentiform, thalamus, and cortex also can cause HCHB. ${ }^{19,23}$ Generally, high attenuation changes involving the contralateral basal ganglia are found on the head CT scan, although in some cases of hyperglycemia they can show a normal result. ${ }^{15,16,17}$ Imaging findings in HCHB due to hyperglycemia are caused by acute infarction, hemorrhagic petechiae, myelinolysis, calcium deposits, and a decrease in aminobutyric synthesis and also acetylcholine due to metabolic changes or the presence of secondary injury due to hyperviscosity or vasogenic edema. ${ }^{20,24}$ The hyperviscosity promotes the disruption of the blood-brain barrier and transient ischemia of striatal neurons. ${ }^{5,20,24}$ The synergistic effect of uncontrolled hyperglycemia and vascular insufficiency promote transient striatum dysfunction, which can result in HCHB. Whereas, in case series about thalamic stroke, evidence that strokes which severe sensory deficit and ataxia are more likely to cause movement disorder. ${ }^{5,13}$ This was in line with our fourth case which had a sensory deficit and there was a lesion at thalamus showed by imaging. 
Table 1. Summary of the cases admission data and clinical courses

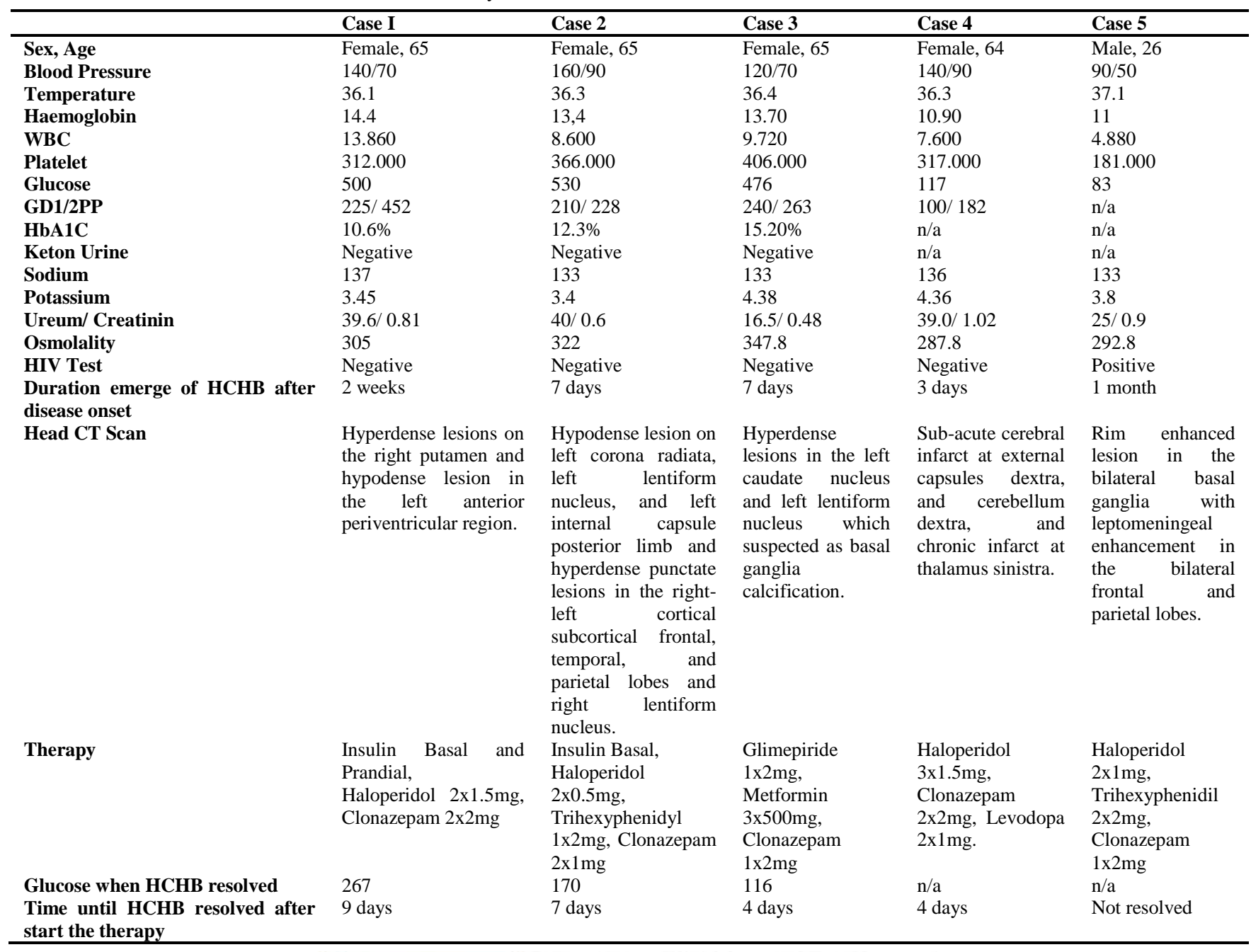

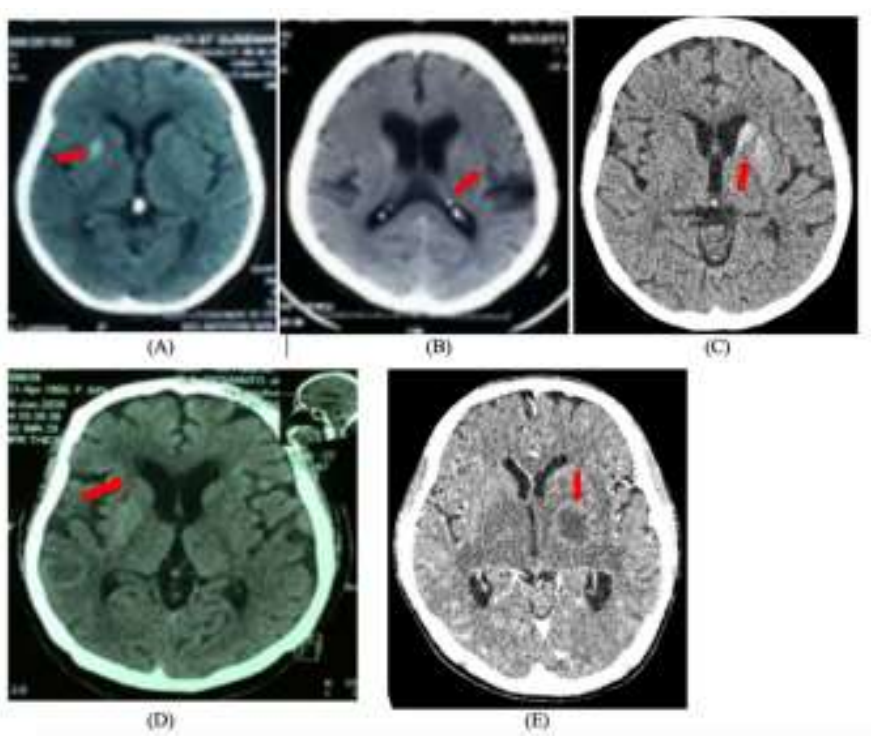

Figure 1. Head CT Scan of cases (A) Hyperdense lesions on the right putamen (Case 1) (B) Hypodense lesion on left corona radiata, left lentiform nucleus (Case 2), (C) Hyperdense lesions in the left caudate nucleus and left lentiform nucleus (Case 3) (D) Hypodense lesion at external capsules dextra, (Case 4) (E) Rim enhanced lesion in the bilateral basal ganglia (Case 5).

Various hypotheses have been proposed to explain HCHB due to hyperglycemia. Hyperglycemia promotes cerebral autoregulation failure, hypoperfusion and activation of anaerobic metabolism, and depletion of GABA in the basal ganglia. Generally, GABA has a role as a main inhibitory neurotransmitter, glutamate as activator neurotransmitter, and acetylcholine as a modulator neurotransmitter. ${ }^{5,17,19}$ Hyperglycemia inactivates the tricarboxylic acid (Krebs) cycle due to decreased regional cerebral blood flow and failure of glucose metabolism, and the brain's metabolism shifts to an anaerobic pathway. ${ }^{16,17,20,21}$ In ketotic condition, acetoacetate can be used to synthesize GABA, whereas, in 
non-ketotic hyperglycemic, changes to anaerobic metabolism promote the brain to use aminobutyric acid, which is synthesized from acetoacetate that causes the decrease of GABA levels rapidly, reduce GABAergic activity, and reduce inhibition of the thalamus by the medial globus pallidus. This condition causes a decrease in inhibitory signals to the thalamus and results in hyperkinetic movements. $5,16,17,18,19,21$

Dopaminergic activity changes in the striatum caused by hyperglycemia are also other mechanisms that can occur, especially in postmenopausal women who lack estrogen.3,21 After menopause, hyperglycemia induces an increase in the sensitivity of dopamine receptors in the striata. Patients HCHB that related to hyperglycemia commonly older women with uncontrolled diabetes and non-ketotic hyperglycemia condition., ${ }^{3,15,21}$

Cerebrovascular insufficiency mechanisms also have a role in HCHB pathophysiology due to hyperglycemia. ${ }^{3,21}$ This condition is similar to the changes that occur in diabetic retinopathy. Hyperglycemia promotes insulin resistance, oxidative stress, and loss of nitric oxide in the endothelium, resulting in endothelial dysfunction and removing endothelial vasoactivators that lead to an increase of free fatty acids, prothrombotic state and dysfunction in smooth vascular muscle and decreased regulation of microRNAs. Vasoconstriction promotes tissue hypoperfusion and neuronal loss or damage. ${ }^{15,17,22}$

Pathophysiology of HCHB due to infection, in this case, cerebral toxoplasmosis, is a tumor-like lesion that causes an increase of intracranial pressure, confusional state, and appearance of focal deficits on the pyramidal pathway. ${ }^{25,26}$ The most common movement disorder in cerebral toxoplasmosis is HCHB or hemichorea-athetosis. Cerebral structures generally affected in the basal ganglia area include the subthalamic nucleus, thalamus, and head of the caudate, putamen, globus pallidus, midbrain, and internal capsule, which can promote contralateral lesions or bilateral movements. ${ }^{9,26}$ HCHB believed to be associated with the subthalamic nucleus damage or its efferent pathways, which eliminates the excitation of the globus pallidus, thereby eliminating the ventral lateral and ventral anterior nuclei that receive projections from the globus pallidus. ${ }^{1,4,9,25}$ In our fifth case, there was rim enhanced lesion on basal ganglia which is believed to be the cause of HCHB on the patient.

HCHB in AIDS patients is commonly associated with the presence of cerebral toxoplasmosis. This is found in $7.4 \%$ of case reports and is generally caused by a subthalamic toxoplasmosis abscess. ${ }^{25,26}$ Some researchers mention that $\mathrm{HCHB}$ that occurs in AIDS patients is a pathognomonic symptom to cerebral toxoplasmosis. ${ }^{25}$ This is interesting as in patients with non-reactive HIV with toxoplasmosis and abscesses in subthalamic, movement disorders are not found. ${ }^{25,27}$

The management principle of HCHB is to determine the etiology and then correct the underlying disorder. ${ }^{6,28,29}$ Pharmacological therapy is not essential in all cases as many patients have a mild and self-limited disorder. HCHB due to hyperglycemia must treat with aggressive glycemic control. The movements will slowly improve after glucose correction. ${ }^{10,27,29,30}$ Patient with acute stroke and HCHB recommends to reduced uncomfortable feeling and distress. $^{22,23}$ Antidopaminergic therapy has the main role of pharmacological treatment, especially a group of typical and atypical neuroleptics and catecholamine-depleting agents. Typical neuroleptic agents including haloperidol, pimozide, perphenazine, and fluphenazine are the first-line drug treatments for $\mathrm{HCHB}$ and work by blocking dopamine receptors. The atypical neuroleptic drugs, such as olanzapine, risperidone, clozapine, quetiapine are less to cause drug-induced parkinsonism and tardive dyskinesia. Clozapine has been successful in refractory cases but can cause agranulocytosis.31 Benzodiazepine drugs including clonazepam and gabapentin often used for additional treatment. Clonazepam suggests using in the short term because of addictive side effects. Levodopa can be considered as a second-line drug of HCHB. Long-term use of levodopa can cause dyskinesia, so the treatment process must be controlled carefully. ${ }^{29,30}$ Management in HCHB due to HIV with toxoplasmosis cerebral includes opportunistic infection treatment, symptomatic treatment of the movement disorder, and the use of antiretroviral agents. $^{25,26,29}$ It has been reported that response to therapy was poor and ineffective in controlling the involuntary movements in the generalized chorea seen with HIV encephalitis. $^{25}$

\section{Conclusion}

Hemichorea-Hemiballism is a rare case of movement disorder. The natural history of hemichorea-hemiballism varies depend on etiology. Characteristic features depend on contralateral disorder within the putamen, caudate nucleus, and globus pallidus. The main management strategy is to determine the etiology and correct the underlying disorder. Prognosis is usually benign in most cases.

\section{Acknowledgement}

The researchers would like to acknowledge Department of Neurology and Brawijaya University for supporting this case reports.

\section{References}

1. Daroff, Robert B, Joseph Jankovic, John C Mazzotta, Scott L Pomeroy. Bradley's neurological in clinically practice. Seventh Edition. USA; 2015.

2. Chang, et al. Chorea-Ballism as a manifestation of decompensated type 2 diabetes mellitus. Am J Med Sci; 2007.333 (3): 175-177.

DOI: 10.1097/MAJ.0b013e3180318e34

3. Lee SH, Shin JA, Kim JH, Son JW, Lee KW, Ko SH, Yang SH, Son BC, Ahn YB. Chorea-ballism associated with nonketotic hyperglycaemia or diabetic ketoacidosis: Characteristics of 25 patients in Korea. Diabetes Research and Clinical Practice; 2011. Aug 1;93(2):e80-3.

4. Chen $\mathrm{C}$, Zheng $\mathrm{H}$, Yang L, Hu Z. Chorea-ballism associated with ketotic hyperglycemia. Neurological Sciences; 2014. Dec 1;35(12):1851-5.

DOI:

$10.1007 / \mathrm{s} 10072-014-1968-1$ 
5. Bizet J, Cooper CJ, Quansah R, Rodriguez E, Teleb M, Hernandez GT. Chorea, hyperglycemia, basal ganglia syndrome $(\mathrm{CH}-\mathrm{BG})$ in an uncontrolled diabetic patient with normal glucose levels on presentation. The American Journal of Case Reports. 2014;15:143. DOI: 10.12659/AJCR.890179

6. Hawley SJ, Weiner WJ. Hemiballismus: Current concepts and review. Parkinsonism and Related Disorders; 2012. 18:125-129.

DOI: $10.1016 /$ j.parkreldis.2011.08.015

7. Bhidayasiri R, Truong DD. Chorea and related disorders. Postgraduate Medical Journal; 2004. Sep 1;80(947):527-34. DOI: 10.1136/pgmj.2004.019356

8. Ohara S. Diabetic Hemichorea-hemiballism. Austin J Clin Neurol; 2015. 2(4):1-6. Avalaible form: https://austinpublishinggroup.com/clinicalneurology/fulltext/ajen-v2-id1037.php

9. Abe Y, Yamamoto T, Soeda T, Kumagai T, Tanno Y, Kubo J, et al. Diabetic striatal disease: Clinical presentation, neuroimaging, and pathology. Intern Med; 2009. 48:1135-41.

DOI: 10.2169/internalmedicine.48.1996

10. Alkilani WZ, Tahir H, Gibb N, Ullah S, Vinod NR. Hyperglycemia induced reversible hemiballismus as the main presentation of newly diagnosed diabetes mellitus. American Journal of Medical Case Reports; 2016. 4(7):245-7. Avalaible from: http://www.anncaserep.com/full-text/accr-v1id1097.php

11. Burn, David. Oxford Textbook of Movement Disorder. Oxford University Press. USA; 2013.

12. Chung SJ, Im JH, Lee MC, Kim JS. Hemichorea after stroke: clinical-radiological correlation. J Neurol 2004; 251: 725-9. DOI: 10.1007/s00415-004-0412-5

13. Cambrea SC, Pascu C, Rugina S. Hemiballismus in a patient with acquired immunodeficiency syndrome and toxoplasma infection. Romanian Journal of Infectious Diseases; 2017. Jan 1;20(1)

14. Cressman S, Rheinboldt M, Lin D, Blasé J. Nonketotic hyperglycemia-induced hemichoreahemiballism. Applied Radiology. 2018 Jun 1;47(6):24-6. Avalaiblr form: https://www.appliedradiology.com/articles/nonketotichyperglycemia-induced-hemichorea-hemiballism

15. Priola AM, Gned D, Veltri A, Priola SM. Case 204: nonketotic hyperglycemia-induced hemiballismhemichorea. Radiology; 2014. Apr;271(1):304-8. DOI: $10.1148 /$ radiol.14120840

16. Kocasoy EO, Atmaca MM, Atmaca M, Hanağasi HA. Chorea-Ballismus Associated with Hyperglycemia. Noro psikiyatri arsivi; 2013. Dec;50(4):375-8. DOI: $10.4274 /$ npa.y6468

17. Alarcón F, Zijlmans JC, Dueñas G, Cevallos N. Poststroke movement disorders: Report of 56 patients. J Neurol Neurosurg Psychiatr; 2004. 75: 1568-1574. DOI: 10.1136/jnnp.2003.011874

18. Borensztein A, Walker RH, Schell R, Guber HA. Hyperglycemia-induced involuntary movements: 2 case reports and a review of the literature. AACE Clinical Case Reports; 2015. Jun;1(3):e165-9

19. Wagner B, Fuhr P, De Marchis GM. Hemichoreahemiballism after acute ischemic stroke. Academia
Journal of Stroke; 2019. Aug 9;1(1). Avalaible from: https://wrightacademia.org/articles/ajs/ajs-1-003.pdf

20. Guo TY, Kuo CC, Chen MH, Lin CI, Chan JS, Hsiao PJ, Shyu HY. Comparison of hemichorea-hemiballism between acute stroke and nonketotic-hyperglycinemia in the clinical scenario: Report of a rare diabetic complication. International Archives of Medicine; 2019. Mar 17;12

21. Chang X, Hong W, Yu H, Yao Y. Chorea associated with nonketotic hyperglycemia: A case report with atypical imaging changes. Medicine; 2017. Nov;96(45). DOI: 10.1097/MD.0000000000008602

22. Ren-Shi $\mathrm{Xu}$. Pathogenesis of diabetic cerebral vascular disease complication. World J Diabetes; 2015. 6(1): 54-66. DOI: 10.4239/wjd.v6.i1.54

23. Handley A, Medcalf P, Hellier K, Dutta D. Movement disorders after stroke. Age Ageing; 2009. 38(3): 2606.

DOI: https://doi.org/10.1093/ageing/afp020

24. McCullen MK, Miller J, Jabbour S, Furlong K, Shirodakar M, Ahmed I, Mandel S. Chorea in the setting of hyperglycemia: A case report and review of the literature. Pract Neurol; 2010. Mar;16-9. Avalaible form: https://practicalneurology.com/articles/2010mar-apr/expert-opinion-chorea-in-the-setting-ofhyperglycemia--a-case-report-and-review-of-theliterature

25. Midi I, Çalişkan HG, Toktaş Z, Sav A, Guenal DI. Generalized choreoathetosis in an acquired immune deficiency syndrome patient with cerebral toxoplasmosis. Turkish Journal of Medical Sciences; 2008. Jul 16;38(4):377-80

26. Rabhi S, Amrani K, Maaroufi M, Khammar Z, Khibri H, Ouazzani M, Berrady R, Tizniti S, Messouak O, Belahsen F, Bono W. Hemichorea-hemiballismus as an initial manifestation in a Moroccan patient with acquired immunodeficiency syndrome and toxoplasma infection: A case report and review of the literature. Pan African Medical Journal; 2011.10.

DOI: $10.4314 /$ pamj.v10i0.72216

27. Tse W, Cersosimo MG, Gracies JM, Morgello S, Olanow CW, Koller W. Movement disorders and AIDS: A review. Parkinsonism Relat Disord; 2004. 10:323-34. DOI: 10.1016/j.parkreldis.2004.03.001

28. Ohara S. Diabetic Hemichorea-hemiballism. Austin J Clin Neurol; 2015. 2(4):1-6. Avalaible form: https://austinpublishinggroup.com/clinicalneurology/fulltext/ajcn-v2-id1037.php

29. Bashir H, Jankovic J. Treatment options for chorea. Expert Review of Neurotherapeutics; 2018 Jan 2;18(1):51-63.

DOI: https://doi.org/10.1080/14737175.2018.1403899

30. Reich, Stephen G, Stewart A. Factor. Therapy of movement disorders a case-based approach. Humana Press. USA; 2019.

31. Herath HM, Pahalagamage SP, Senanayake S. Case report of hyperglycemic nonketotic chorea with rapid radiological resolution. BMC Medical Imaging; 2017. Dec $1 ; 17(1): 54$ Avalaible from: https://bmcmedimaging.biomedcentral.com/articles/10 .1186/s12880-017-0228-2 